\title{
Paying less but harvesting more: the effect of unconscious acceptance in regulating frustrating emotion
}

\author{
DING NanXiang ${ }^{\dagger}$, YANG JieMin ${ }^{\dagger}$, LIU Ying Ying \& YUAN JiaJin ${ }^{*}$ \\ Key Laboratory of Cognition and Personality, Ministry of Education, Faculty of Psychology, Southwest University, Chongqing 400715, China
}

Received April 13, 2015; accepted April 27, 2015; published online June 26, 2015

\begin{abstract}
Previous studies indicate that emotion regulation may occur unconsciously, without the cost of cognitive effort, while conscious acceptance may enhance negative experiences despite having potential long-term health benefits. Thus, it is important to overcome this weakness to boost the efficacy of the acceptance strategy in negative emotion regulation. As unconscious regulation occurs with little cost of cognitive resources, the current study hypothesizes that unconscious acceptance regulates the emotional consequence of negative events more effectively than does conscious acceptance. Subjects were randomly assigned to conscious acceptance, unconscious acceptance and no-regulation conditions. A frustrating arithmetic task was used to induce negative emotion. Emotional experiences were assessed on the Positive Affect and Negative Affect Scale while emotion-related physiological activation was assessed by heart-rate reactivity. Results showed that conscious acceptance had a significant negative affective consequence, which was absent during unconscious acceptance. That is, unconscious acceptance was linked with little reduction of positive affect during the experience of frustration, while this reduction was prominent in the control and conscious acceptance groups. Instructed, conscious acceptance resulted in a greater reduction of positive affect than found for the control group. In addition, both conscious and unconscious acceptance strategies significantly decreased emotion-related heart-rate activity (to a similar extent) in comparison with the control condition. Moreover, heart-rate reactivity was positively correlated with negative affect and negatively correlated with positive affect during the frustration phase relative to the baseline phase, in both the control and unconscious acceptance groups. Thus, unconscious acceptance not only reduces emotion-related physiological activity but also better protects mood stability compared with conscious acceptance. This suggests that the clinical practice of acceptance therapy may need to consider using the unconscious priming of an accepting attitude, instead of intentionally instructing people to implement such a strategy, to boost the efficacy of acceptance in emotion regulation.
\end{abstract}

unconscious acceptance, emotion regulation, frustration, heart-rates, positive affect, negative affect

Citation: Ding NX, Yang JM, Liu YY, Yuan JJ. Paying less but harvesting more: the effect of unconscious acceptance in regulating frustrating emotion. Sci China Life Sci, 2015, 58: 799-809, doi: 10.1007/s11427-015-4875-7

In daily life, people often encounter all kinds of negative events. The negative emotion induced by these events needs to be regulated to avoid interference with ongoing activities, situational demands or long-term goals. Successful control of emotional impulses is important for an individual's well-being, health and social functioning [1-4].

$\dagger$ Contributed equally to this work

*Corresponding author (email: yuanjiaj@swu.edu.cn)
Inspired by Gross's process model of emotion regulation, prior studies have proposed two well-known strategies: cognitive reappraisal and expressive suppression [5-7]. Cognitive reappraisal focuses on the changing of the internal interpretation of stimulus meanings, while expression suppression focuses on the changing of the fully blown emotional responses [5]. Different from these traditional regulation strategies, acceptance is an exposure-based strategy defined as "the aware embracing of emotional 
events and the active experiencing of their emotional consequences, without attempts to change the frequency, form or impacts of the emotional events" [8]. In clinical practice, a number of psychotherapies based on acceptance have been developed, such as acceptance and commitment therapy [9-11], mindfulness-based cognitive therapy [12,13], and acceptance-based behavioral therapy $[14,15]$. The essence of these therapies is to accept emotional events and their emotional impact, such as training patients to fully experience their own internal and external feelings with a non-judgment attitude, and highlighting attention devotion to more valuable aspects of life. Clinical results indicate that psychotherapies based on acceptance are successful in the treatment of anxiety and depression $[8,16]$. The laboratory study of acceptance also found positive effects of acceptance, including lower negative emotion intensity [17-19], the reduction of anxiety and depression [20-22], and better adaptability to chronic pain [23]. Recently, Shallcross et al. [24] suggested that accepting negative emotion prevents individuals from long-term involvement in a negative mood and avoids negative emotion developing into depression.

However, all the above strategies require subjective efforts mobilizing cognitive resources to achieve the purpose of emotion regulation. This is also true for the application of unconscious acceptance, which requires people to accept or embrace unpleasant events and emotional consequences that are naturally processed with defensive motivation [25]. This process, which is counter to natural tendencies, is thus most likely resource costly [26]. This may explain why there is also evidence showing that the acceptance strategy is associated with increases rather than decreases in negative feelings [27,28], or produces no beneficial effect, similar to rumination [29]. Although most current research focuses on conscious emotion regulation, some research has indicated that implicit (largely unconscious) processes are strongly involved in emotion regulation [30]. Compared with unconscious emotion regulation, conscious emotion regulation needs more cognitive resources [31-33]. For instance, it has been consistently reported that the conscious suppression of negative emotion enhances sympathetic physiological activations, disrupts social and cognitive dysfunctions [34], or even impairs physical health [31-33]. These adverse outcomes, however, are absent when unconscious emotion regulation is used. For example, previous studies have indicated that priming unconscious regulation goals decreases experienced anger without maladaptive physiological consequences [35], and an automatic attention shift from negative to positive cues accounts for the enhanced positive affects in older people $[30,36,37]$. As other unconscious processes [38-41], unconscious regulation may occur without subjective awareness and cost little in terms of the attention resource or subjective effort.

On the basis of the evidence described above, we propose that it is more adaptive to regulate emotions implicitly.
Koole and Rothermund [42] defined implicit emotion regulation as the process of modifying the quality, intensity, or duration of emotional responses without the need for conscious supervision and explicit intentions. The implicit emotion regulation is often realized by the unconscious pursuit of a goal. For this purpose, researchers have preceded the emotion-evoking task with an unconscious priming procedure. For instance, Mauss et al. [35] found that unobtrusively priming people with emotional control words (e.g., cool, covered) led to a less angry experience in response to a laboratory anger provocation compared with people primed with words related to emotion expressions (e.g., volatile, boiled). Additionally, Gallo and colleagues [43] showed that automatizing emotion regulation goals by an implementation practice reduces the reactivity of the negative affect to mutilation images in disgust-sensitive individuals and to fearful images in spider-fearful individuals, though reducing negative affects was not explicitly requested. Additionally, a recent study conducted in our laboratory revealed lower frustration-related heart-rate reactivity when reappraisal was unconsciously primed by a sentence unscrambling task than when no priming was received [44]. Recently, it was found that, as an important way of priming implicit regulation, creating implementation intention alone (e.g., if I see a dog approaching me, then I will stay calm) is sufficient to change emotion-relevant behaviors even in the absence of implementation practices [45].

Thus, the present study aimed at comparing the effects of emotion regulation using conscious and unconscious acceptance strategies, with affective responses measured in both behavioral (subjective emotion rating) and physiological (heart rate) levels. The heart rate was used as the physiological index of emotion regulation, because this index has been verified to be sensitive to frustration [46], and changes in emotion arousal [47].

Although many studies have assessed the effects of emotion regulation in the context of viewing emotional scenes or film clips $[43,48]$, negative emotion in real-life settings often comes from frustrations and setbacks, such as failing an exam, losing a game or being unable to accomplish predetermined goals. Thus, it is practically important to assess the effects of negative emotion regulation in ecologically valid, frustrating situations. With this consideration, the present study manipulated a complex, frustrating arithmetic task to induce negative emotions.

Williams et al. [47] found that priming subjects with reappraisal-relevant words, despite lacking overt reappraisal instructions, effectively reduced cardiovascular responses. Similarly, we predict that priming subjects with acceptance-relevant words is able to reduce subjects' affective response in terms of behavioral or physiological levels, and this regulation effect may be more pronounced during unconscious relative to conscious acceptance, because the instructed, conscious acceptance is resource costly and may enhance temporary negative feelings [26-28]. Furthermore, 
as a physiological measure such as the heart rate has been validated as an index of frustration, with the heart rate increasing with frustration $[46,49]$, we predict that heart-rate measures in the current study would be positively correlated with the experienced negative emotion during the frustrating task.

\section{Method}

\subsection{Participants}

Fifty-nine undergraduates (31 males; $\bar{x}=21.7 ; \mathrm{SD}=5.1$ ) were recruited for the study and were randomly divided into three groups: a control group $(N=20,8 \mathrm{~F} / 12 \mathrm{M})$, conscious acceptance group $(N=20,11 \mathrm{~F} / 9 \mathrm{M})$, and unconscious acceptance group $(N=19,9 \mathrm{~F} / 10 \mathrm{M})$. Participants were paid 20 RMB at the end of the experiment. The experimental session lasted approximately $60 \mathrm{~min}$. All the subjects reported no history of affective disorder and were free of any psychiatric medication. The subjects were free of anxiety or depression symptoms, as indicated by relatively low scores for the measures of the Spielberg State/Trait Anxiety Scale [50] and Beck Depression Inventory II [51]. The subjects were right-handed and had normal or corrected-to-normal vision.

To verify whether our randomization was successful, we tested whether the three groups were similar in emotional states, emotion-related personality traits and gender composite. There were no significant group differences in emotion-related states and personality traits, indicated by similar scores in the Spielberg State Anxiety Scale $(F(2,56)=0.71$, $P=0.50)$, the Spielberg Trait Anxiety Scale $(F(2,56)=1.01$,
$P=0.35)$, the Beck Depression Inventory-II $(F(2,56)=1.37$, $P=0.26)$ and the Rosenberg Self-Esteem Scale [52] $(F(2$, $56)=2.19, P=0.12$; Figure 1$)$. In addition, the gender composite was not significantly different across the three groups; $\chi^{2}(2,57)=0.983$. The experiment used the Emotion Regulation Questionnaire [32] and Acceptance and Action Questionnaire [53] to check whether the habitual emotion regulation style was successfully controlled. The results showed no significant differences in the habitual use of suppression $(F(2,56)=1.99, P=0.15)$, reappraisal $(F(2,56)=1.25, P=0.29)$ and acceptance $(F(2,56)=0.67, P=0.52)$ (Figure 1).

\subsection{Frustrating task}

The frustrating task included 20 trials. In each trial, participants were instructed to count the number of triangles in a complex graphic presented on the computer screen. They received feedback of whether their answer was correct or incorrect. Incorrect feedback and correct feedback appeared in a fixed order, but this manipulation was unknown to the subjects. Correct feedback was presented in the second and seventh trials and incorrect feedback in the other trials. The 20 graphics used for the task were selected from 38 graphics that were evaluated in a pilot study. A separate group of 40 participants took part in this pilot to evaluate the material. After counting the number of triangles in the complex figure, participants were instructed to rate the complexity of the figure on a scale from 1 (not difficult at all) to 8 (extremely difficult). Their response times during triangle counting were also recorded. On the basis of these results, we selected 18 moderately complex figures, for which the

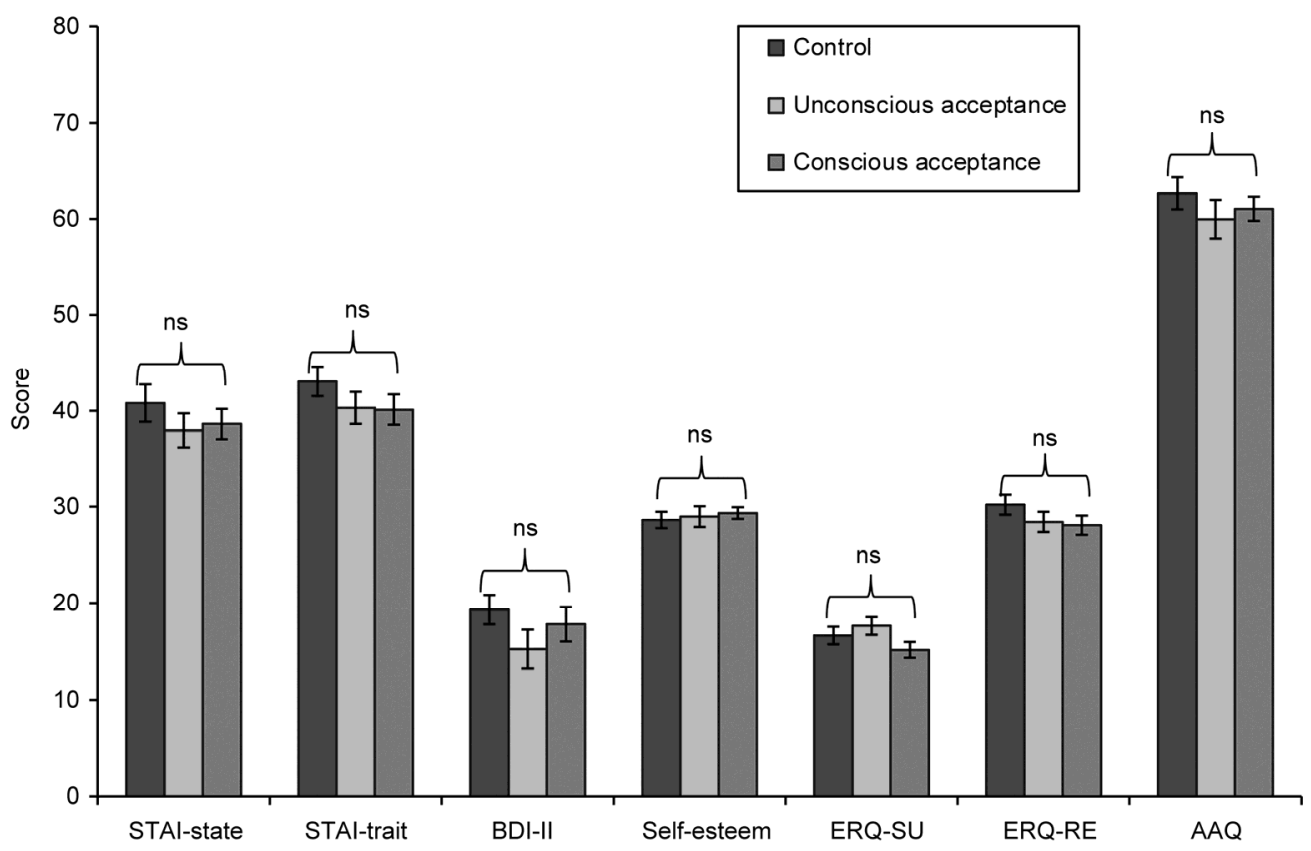

Figure 1 The scores of three groups on the Spielberg State Anxiety Scale, Spielberg Trait Anxiety Scale, Beck Depression Inventory-II, Rosenberg Self-Esteem, ERQ-RE (reappraisal), ERQ-SU (suppression) and AAQ (Acceptance and Action Questionnaire). Error bars represent the standard errors of the mean values. ns: not significant. 
averaged response time was $39 \mathrm{~s}$.

To ensure that participants were not skeptical of the experimental purpose, the 18 graphics associated with erroneous feedback were moderately difficult and the two graphics associated with correct feedback were relatively simple. At the same time, according to the average time that participants spent on the 18 moderately complex figures in the material assessment, the maximum duration of figure presentation was set to $39 \mathrm{~s}$ in the experiment.

\subsection{Procedure}

After arrival at the laboratory, participants were placed in a separate room containing the psychophysiological recording equipment, where they received information about the triangle-counting task. Participants were then prepared for psychophysiological recordings. We attached a sensor to each participant's thumb for continuous recording of the heart rate. The experiment consisted of three phases: a baseline phase, a task phase and a recovery phase.

\subsubsection{Baseline phase}

In the baseline phase, we instructed participants to rest for 3 min, during which time the baseline heart-rate reactivity was recorded. After this 3-min rest time, participants were asked to complete the Positive Affect and Negative Affect Scale (PANAS). This phase was designed to control the potential effect of pre-experiment group differences on the emotion regulation effects.

\subsubsection{Task phase and emotion regulation instruction}

In the task phase, participants performed two tasks. First, they were required to complete a scrambled sentence task, in which they needed to construct a grammatical four-word sentence from five-word jumbles [54]. Those primed with the acceptance goal unscrambled five neutral sentences and 10 sentences containing words or phrases related to acceptance (such as "accept"). Participants in the conscious reappraisal and control groups unscrambled 15 neutral sentences.

In the second task, participants were given an explanation of the difficult triangle-counting task. The instructions were: "First, the screen will present a ' + ' fixation alerting you to begin the test. After the ' + ' fixation disappears, a complex figure will appear on the screen, the figure will be presented for $39 \mathrm{~s}$ (the average completion time for college students in our previous study). Please count the number of triangles embedded in the figure within this time limit, and input your answer after the figure disappears. Once inputting the answer, feedback about the correctness of your answer will be provided for $300 \mathrm{~ms}$. If you have understood the instructions, press the 'Enter' key to start the experiment." Unknown to the subjects, the feedback was manipulated to be negative in 18 trials ("wrong") and to be positive in two trials (correct). Final feedback was provided for $30 \mathrm{~s}$ at the end of the experiment, which reminded subjects of their overall performance in the task (10\%; i.e., 2/20).

Participants under the unconscious acceptance and control conditions received no further instructions. Participants under the conscious acceptance condition were provided with the following additional instructions [27]: "The task is a bit difficult and therefore you may sometimes experience frustration. If this happens, please try to accept and experience your negative emotion naturally and not to change or control it in any way. Let your emotion run naturally, and think of it as a natural phenomenon, just like a cloud passing in the sky. Please allow yourself to remain harmonious with your negative emotions."

After participants completed the frustrating task, the feedback about the accuracy (10\%) of triangle counting was presented on the screen. Participants then rated their emotional experience again. Additionally, the conscious acceptance group needed to rate the extent to which they applied the emotion regulation instructions during the task.

\subsubsection{Recovery phase}

After completing the frustrating task, a 3-min rest (recovery phase) was implemented; this phase was designed to help participants recover from the negative mood induced by the frustrating task. Participants completed the modified PANAS questionnaire when the recovery was over.

Finally, participants were probed for suspicion of the experimental purposes and debriefed using the funneled debriefing method [55]. No participants indicated suspicion of the purposes of the priming and task procedures.

\subsection{Behavior and physiological measures}

\subsubsection{Subjective emotion experience}

Emotion experience was assessed using PANAS [56]. PANAS contains 20 emotion descriptors, 10 items measuring positive affect and 10 items measuring negative affect. All items in PANAS are typically rated on five-point Likert scales, ranging from 1 (not at all) to 5 (extremely). To measure emotion changes in participants more sensitively, all items were rated on seven-point Likert scales, ranging from 1 (not at all) to 7 (extremely), in our experiment. In the experiment, all the items of PANAS were encoded into the E-prime program. Participants press the corresponding number key to complete the rating.

\subsubsection{Physiological measure}

In the experiment, we used a Spirit biofeedback instrument with 10 wireless telemetry (Bluetooth) channels. Physiological channels were sampled at $32 \mathrm{~Hz}$. Biotrace software recorded the data simultaneously with the experimental progress. The heart rate was used as a measure of physiological activity related to the emotional response, consistent with recent investigations of the effect of emotion regulation on cardiovascular reactivity $[35,47]$. During the collec- 
tion of data, the biotrace software computed average values in each phase.

\section{Results}

\subsection{Manipulation check}

The first manipulation check was to examine whether our triangle-counting task successfully induced negative emotion. We conducted a paired-sample $t$-test of subjective experience and heart-rate measures between baseline and task phases in the control group, where emotion induction was free of regulatory influences. The positive emotion measure of PANAS was significantly lower in the task phase $(\bar{x}=35.20, \mathrm{SD}=8.77)$ than in the baseline phase $(\bar{x}=42.90$, $\mathrm{SD}=8.93 ; t(19)=3.9, \quad P<0.001)$. The negative emotion measure of PANAS was significantly higher in the task phase $(\bar{x}=26.75, \mathrm{SD}=8.83$ ) than in the baseline phase $(\bar{x}=16.85, \mathrm{SD}=4.72 ; t(19)=-5.2, \mathrm{p}<0.01)$. The heart-rate reactivity was significantly higher in the task phase ( $\bar{x}=86.30, \mathrm{SD}=9.45)$ than in the baseline $(\bar{x}=82.0$, $\mathrm{SD}=9.72 ; t(19)=-5.4, P<0.001)$. These results verified that the triangle-counting task used in this study successfully induced negative emotional consequences.

The statistical analysis of the change scores of subjective emotion and heartbeats from baseline to frustration phases also supported that the frustration-inducing task successfully induced a negative emotional state. We compared the change scores of positive affect, negative affect, and heartbeat measures with a score of zero in the control group. Results showed that the positive affect measure $(t(19)=$ $-4.03, \quad P<0.01)$, negative affect measure $(t(19)=4.73$, $P<0.01)$ and heartbeat measure $(t(19)=5.18, P<0.01)$ were all significantly different from zero.

The second manipulation check examined whether subjects under the conscious acceptance condition successfully complied with the acceptance instruction. Subjects in the conscious acceptance group were asked to rate the extent to which they accepted their frustration on a six-point scale (1: not at all; 6: extremely) immediately after completing the task. The analysis of the instruction confirmation ratings showed that the acceptance strategy was successfully used in the task $(\bar{x}=4.38, \mathrm{SD}=0.70)$. The scores were significantly higher than the midpoint of the rating scale $(t(20)=8.55, P<0.01)$.

The third manipulation check aimed to examine whether the time spent on the frustrating task, and the time interval from the task termination to the start of the recovery period, were similar across the three groups. Results showed that the control group ( $\bar{x}=975 \mathrm{~s}, \mathrm{SD}=19 \mathrm{~s})$, conscious acceptance group ( $\bar{x}=978 \mathrm{~s}, \mathrm{SD}=21 \mathrm{~s}$ ) and unconscious acceptance group ( $\bar{x}=970 \mathrm{~s}, \mathrm{SD}=22 \mathrm{~s}$ ) had no significant difference in the time spent on the frustrating task $(F(2,56)$ $\left.=0.75, P=0.48, \eta^{2}=0.03\right)$. Additionally, the control group
( $\bar{x}=33.70 \mathrm{~s}, \mathrm{SD}=2.77 \mathrm{~s}$ ), conscious acceptance group $(\bar{x}=34.65 \mathrm{~s}, \mathrm{SD}=2.34 \mathrm{~s})$ and unconscious acceptance group ( $\bar{x}=33.79 \mathrm{~s}, \mathrm{SD}=2.62 \mathrm{~s}$ ) had no significant difference in the time interval from the end of the frustrating task to the beginning of the recovery period $(F(2,56)=0.82, P=0.45$, $\left.\eta^{2}=0.03\right)$.

\subsection{Randomization check}

Analyses of variance (ANOVAs) were conducted to examine whether the experimental groups were similar in terms of their baseline emotional state. The three groups showed similar levels of positive emotion (PANAS-P; $F(2$, 56) $=0.34, P=0.72, \eta^{2}=0.01$ ), negative emotion (PANAS-N; $\left.F(2,56)=0.38, P=0.68, \eta^{2}=0.013\right)$ and heart rate $(F(2$, 56)=2.33, $\left.P=0.11, \eta^{2}=0.077\right)$. Thus, the three groups were similar in their pre-experiment emotional state, ruling out the possibility that post-experiment group differences were due to pre-treatment group differences in emotional states.

\subsection{Emotion regulation effect on the subjective expe- rience}

To check whether different experimental treatments have a significant effect on the individual's emotional level during the frustration-inducing phase, we conducted univariate ANOVAs with the positive and negative emotional experiences during the frustrating task (Figure 2). The emotional level was measured using the positive affect index and negative affect index of PANAS. First, we conducted ANOVA with the negative affect index of PANAS. To control the possible effect of individual differences in the baseline phase, the negative affect in the baseline phase was entered as a covariate. Results revealed that there was no significant difference among the three groups; $F(2,55)=0.86, P=0.43$, $\eta^{2}=0.030$. Similarly, we conducted ANOVA with the positive affect index of PANAS, and the positive affect index in the baseline phase was entered as a covariate. The ANOVA revealed that the three groups were significantly different in terms of their positive affect index during the experience of frustration; $F(2,55)=4.2, P=0.02, \eta^{2}=0.134$. Post hoc comparisons using Bonferroni correction indicated that the unconscious acceptance group ( $\bar{x}=38.42, \mathrm{SD}=9.01$ ) was significantly different from the conscious acceptance group $\left(\bar{x}=32.80, \mathrm{SD}=10.03 ; \quad F(1,36)=8.09, P=0.02, \eta^{2}=0.18\right)$. There were no significant differences between the unconscious acceptance group and the control group $(\bar{x}=35.20$, $\left.\mathrm{SD}=8.78 ; F(1,36)=2.91, P=0.41, \eta^{2}=0.08\right)$ or between the conscious acceptance group and the control group $(F(1$, $\left.37)=1.68, P=0.48, \eta^{2}=0.04\right)$. To control for the possible effect of individual differences in emotion-related traits on the group differences of interest, we analyzed positive affect changes (frustration-baseline) using SATI-state, SATI-trait, BDI, Rosenberg Self-esteem, ERQ-RE, ERQ-SU and AAQ 
scores as covariates. The results showed greater positive affect reduction for the conscious acceptance group $(\bar{x}=-11.71)$ than for the unconscious $(\bar{x}=-2.07, P=0.001)$ and control ( $\bar{x}=-6.62, P=0.05)$ groups; while the positive affect reduction tended to be less for the unconscious group than for the control group $(P=0.088)$. Additionally, the direct test of the frustration-baseline differences showed a significant positive affect reduction in the conscious $(P<0.001)$ and control $(P=0.001)$ groups but not in the unconscious group $(P=0.27)$, indicated by a significant phaseby-group interaction $\left(F(2,49)=6.69, P=0.003, \eta^{2}=0.21\right)$.

To check whether the emotional recovery procedure effectively reduced negative consequences elicited by the frustrating situation, we conducted a $t$-test of the subjective affect indexes of the three groups from frustration to recovery phases. The negative affect report was significantly less in the recovery phase than in the frustration phase for each group; $P<0.01$. In addition, the positive affect reported by the conscious acceptance group was significantly higher in the recovery phase than in the frustration phase; $t(19)=-2.3$, $P=0.04$. These results suggest that the recovery procedure was useful in helping participants to recover their mood. To further assess the degree of emotional recovery of participants, we compared the emotional measures between baseline and recovery phases. The negative affect was not significantly different between recovery and baseline phases for all three groups $(P>0.05)$, and the positive affect index of the three groups was significantly lower in the recovery phase than in the baseline phase; $P<0.01$. The results reveal that, though the recovery procedure was useful in restoring the mood state, it did not restore the subjects' mood to the baseline level. This further suggests that the frustrating situation successfully induced a negative emotional consequence.
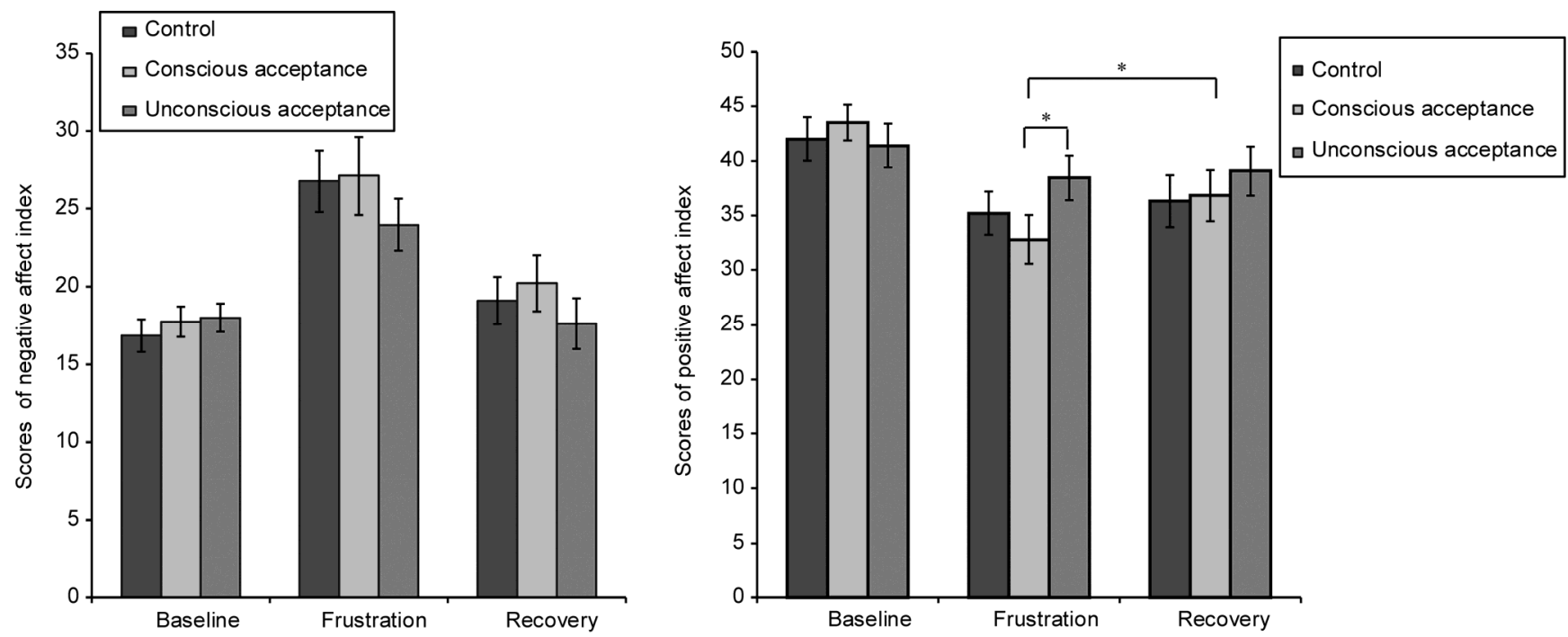

\subsection{Emotion regulation effect on the physiological index}

For physiological activity, a similar ANOVA was conducted with the heart-rate activity during the task. To control for the possible effect of individual differences in baseline heart-rate reactivity, the heart rate in the baseline phase was entered as a covariate. The ANOVA revealed that the three groups were significantly different in terms of their heart-rate reactivity; $F(2,55)=4.31, P=0.02, \eta^{2}=0.135$ (Figure 3 ). Post hoc comparisons using Bonferroni correction indicate that the unconscious acceptance group $(\bar{x}=82.72$, $\mathrm{SD}=9.63$ ) had significantly lower heart-rate reactivity than the control group ( $\bar{x}=86.30, \mathrm{SD}=9.45 ; \quad F(1,36)=7.40$, $\left.P=0.01, \eta^{2}=0.17\right)$ during the experience of frustration. In addition, the conscious acceptance group ( $\bar{x}=78.29$, $\mathrm{SD}=7.69$ ) had significantly lower heart-rate reactivity than the control group $(\bar{x}=86.30, \mathrm{SD}=9.45 ; F(1,36)=4.37$, $\left.P=0.03, \eta^{2}=0.11\right)$. However, the unconscious acceptance and conscious acceptance groups showed no significant differences in their heart-rate reactivity during the experience of frustration $\left(F(1,36)=0.01, P=0.92, \eta^{2}<0.01\right)$. These results were reliable, as the same group differences were obtained after entering emotion-related personality measures as covariates into the ANOVA model; $F(2$, 48)=3.89, $P=0.03, \eta^{2}=0.14$.

Similarly, to check whether the recovery procedure effectively reduced physiological activity elicited by frustration, we compared the heart-rate reactivity of the three groups during the recovery phase with that during the frustration phase. The heart-rate reactivity of each of the three groups was significantly lower in the recovery phase than in the frustration phase; $P<0.01$. To further assess the extent of mood recovery, we conducted a $t$-test with the heart-rate reactivity in baseline and recovery phases. The heart-rate reactivity of each of the three groups showed no significant

Figure 2 Mean subjective emotion ratings for baseline, task (frustration) and recovery phases in the three groups. Error bars represent the standard errors of the mean values. $*$ : $P<0.05$. 


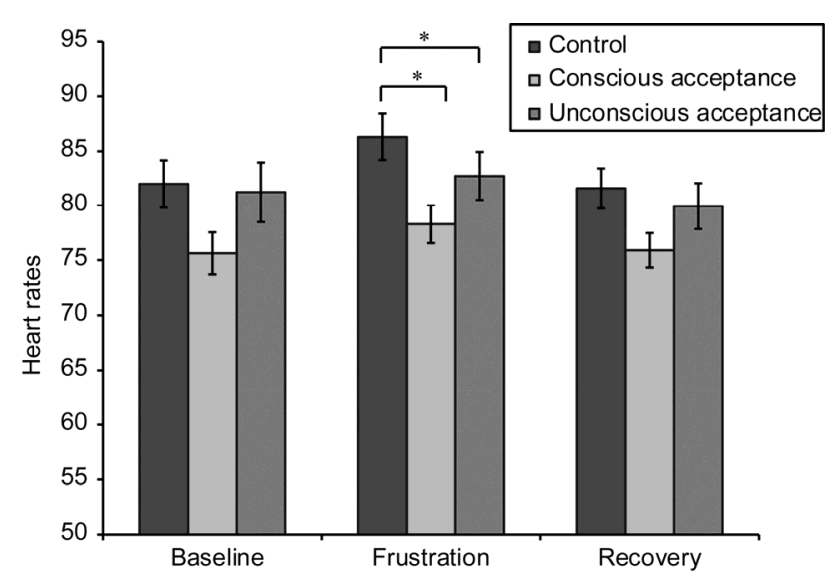

Figure 3 Mean heart rates for pre-experiment baseline, task (frustration) and recovery phases in the three groups. Error bars represent the standard errors of the mean values. *: $P<0.05$.

differences between these two phases. The result suggests that the recovery procedure was useful in restoring physiological activity to the pre-experiment state for each group.

\subsection{Correlations between subjective emotion and heart- rate activity}

To examine whether the enhanced heart-rate reactivity dur-
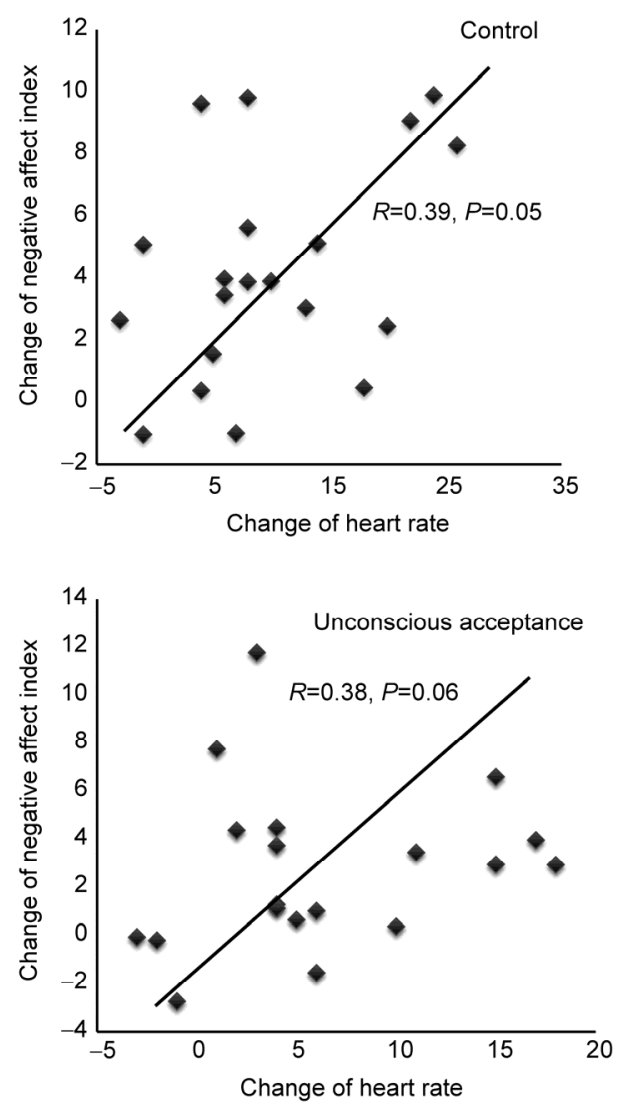

ing the frustrating task effectively predicts an increase in the level of the negative subjective experience, we analyzed the Spearman correlation between the reported emotional change and the heart-rate change during the frustration phase relative to the baseline phase in the control group. At the same time, to further explore the regulating effects of conscious acceptance and unconscious acceptance, we conducted similar correlation analyses for the conscious acceptance and unconscious acceptance groups (Figure 4). The results showed a positive correlation between the negative affect change and heart-rate change $(R=0.39, P=0.05)$ and a trend of negative correlation between positive affect change and heart-rate change $(R=-0.31, P=0.09)$ in the control group. Similarly, there was a trend of positive correlation between the negative affect change and heart-rate change $(R=0.38, P=0.06)$ and a trend of negative correlation between the positive affect change and heart-rate change $(R=-0.33, P=0.08)$ in the unconscious acceptance group. However, there was no correlation between the negative affect change and heart-rate change $(R=0.00, P=0.50)$ or between the positive affect change and heart-rate change $(R=0.10, P=0.33)$ in the conscious acceptance group. These results suggest that heart-rate activity is a likely valid predictor of negative emotion intensity in unconscious acceptance and control groups.
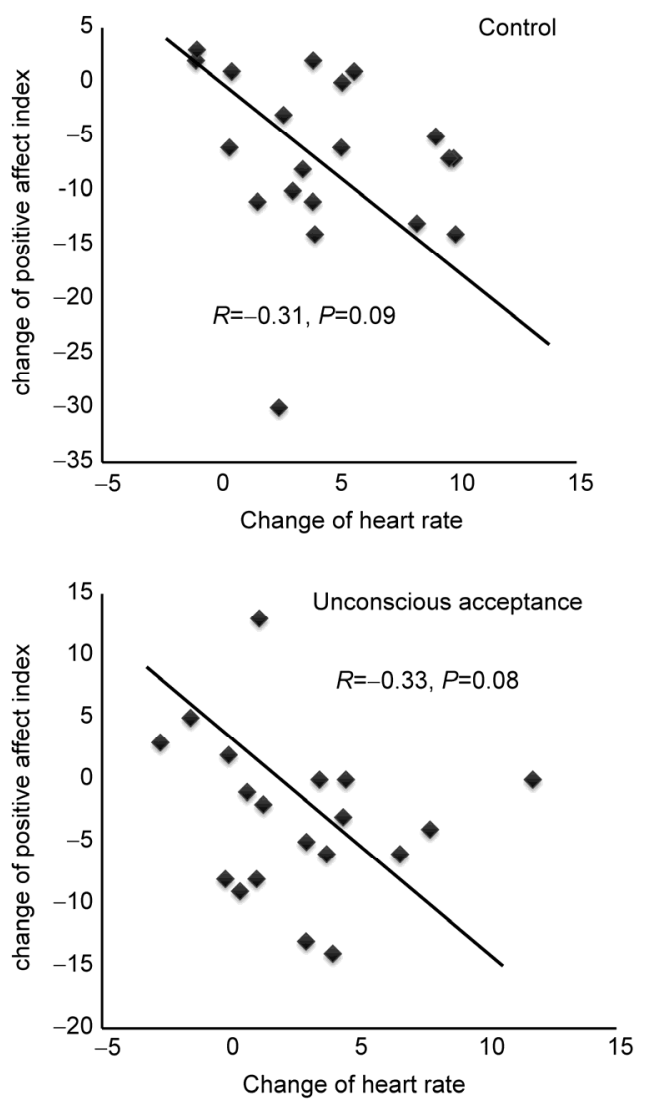

Figure 4 Scatterplots for the correlations between PANAS and heart-rate reactivity during the frustration phase relative to the baseline phase in the control (top panel) and unconscious acceptance (bottom panel) groups. 


\section{Discussion}

Previous studies suggest that personal relevance is an important component for effective emotional induction, such that emotional stimuli with personal relevance evoked more intense emotional reactivity than those without personal relevance [57,58]. In real-life settings, negative emotions that people experience often result from negative events that have close personal relevance, such as self-experienced frustrations and setbacks like failing an exam or losing a sports game. Both this empirical evidence and anecdotal evidence imply that it is highly important to induce emotion in an ecologically valid, self-relevant paradigm. Although many prior studies have assessed the effects of emotion regulation in the context of viewing emotional pictures or film clips $[17,18,27,59,60]$, the depictions of these materials were not directly relevant to the observers. To date, few studies have used experimental paradigms whose emotion induction closely resembles that in natural situations, such as negative emotions induced by frustration and setbacks. On the basis of these considerations, the present study manipulated an ecologically valid, frustrating arithmetic task to induce negative emotions and investigate emotion downregulation by unconscious acceptance. The results showed that unconscious acceptance produced little reduction of positive affect, while such reduction was most pronounced during conscious acceptance of frustration. In addition, both conscious and unconscious acceptance strategies significantly decreased emotion-related heart-rate activity (to a similar extent) in comparison with the control condition. In the control and unconscious acceptance groups, heart-rate reactivity was positively correlated with the negative affect rating and negatively correlated with the positive affect rating during the frustration phase compared with the baseline phase. However, heart-rate reactivity had no correlation with positive and negative affect ratings in the conscious acceptance group. These results suggest that unconscious acceptance is more effective than conscious acceptance in regulating negative emotional consequences during the experience of frustration.

Many researches have shown that conscious acceptance effectively reduces negative emotion and its relevant physiological arousal $[18,61,62]$. Consistent with this evidence, the present study found that conscious acceptance effectively reduced subjects' frustration-related heart rate activity. However, the instructed, conscious acceptance did not reduce, and significantly increased, the subjective frustrating experience in comparison with the control group, as shown by the largest positive affect reduction in the conscious acceptance group during the frustrating task (Figure 2). This is consistent with existing evidence showing that instructed acceptance is unable to decrease negative emotion immediately after emotion induction [27-29,63]. In addition, consciously instructed acceptance requires subjects to embrace their negative emotions naturally and even to experience the feeling deeply $[14,15]$. Fully embracing negative experi- ences requires intentional dropping of the natural defensive tendencies. This process is resource costly and may intensify immediate unpleasant feelings [28]. This most likely explains why instructed acceptance was associated with the lowest positive affect values. Although conscious acceptance reduced frustration-related physiological reactions, it did not reduce subjective experienced frustration. The dissociation of subjective emotional and physiological indexes during conscious acceptance was also supported by the lack of a correlation between the heart rate and emotional experiences during conscious acceptance. In addition, some studies have suggested that conscious acceptance is unable to regulate emotional feeling immediately after the subject experiences stress [28], watches annoying videos or recalls a disaster experience [27]. Clinical studies on acceptance found that the outcome of mindfulness therapies based on acceptance may emerge after a long period of training $[64,65]$. During the recovery stage of our study, the positive affect index of the conscious acceptance group showed a notable improvement, suggesting that the regulation of emotional feeling using conscious acceptance may not be an immediate, fast process.

The current study found that, in contrast to conscious acceptance, unconscious priming of the acceptance strategy was not only useful in maintaining subjects' positive affective state but also significantly mitigated emotion-related physiological activity during the experience of frustration. A couple of previous studies indicated that unconscious regulatory processes reduced negative emotional experiences or emotion-related physiological reactivity, without the cost of cognitive effort or physiological resources $[30,35,37]$. In the present experiment, unconscious acceptance effectively regulated heart-rate reactivity during the experience of frustration. Consistent with our result, Williams and colleagues [47] observed that unconscious reappraisal also successfully reduced heart-rate reactivity elicited by anxiety. For the subjective emotional index that was not investigated in Williams' study, we observed that unconscious acceptance was linked with the smallest, non-significant reduction of positive affect measures. In the unconscious acceptance group, heart-rate reactivity was positively correlated with the negative affect rating and negatively correlated with the positive affect rating during the frustration phase compared with the baseline phase. These results showed that, unlike conscious acceptance, unconscious acceptance did not lead to a dissociation of subjective experience and physiological reactivity, probably because unconscious acceptance does not entail the intentional, conscious embrace of frustrating, unpleasant emotional consequences. Therefore, compared with conscious acceptance, unconscious acceptance is more adaptive in regulating frustrating emotional consequences.

Unlike the regulation effect on the positive affect index, conscious acceptance and unconscious acceptance had no significant difference in terms of the regulation effect on the 
negative affect index. There may be two potential reasons for this. Firstly, the PA (positive affect) and NA (negative affect) subscales of PANAS are two relatively independent dimensions $[66,67]$. PA reflects the extent to which a person feels enthusiastic, active, and alert; NA reflects the extent to which a person feels distress, displeasure, and disgust [56]. There is evidence showing that PA and NA only have low to moderate negative correlation [56]. This suggests that, though a decline in the PA score might accompany a rise in the NA score, this relation is not robust, and may vary across different situations. Secondly, PA and NA are different in terms of the emotional categories they are sensitive to. For example, studies have found that PA is different from NA in its efficacy for discerning clinical mood disorders: it is difficult to discern anxiety from depression by the use of the NA index, while the PA index is a valid index of depression but has little correlation with anxiety [68,69]. This suggests that PA, instead of NA, is an effective scale for assessing depression. Crawford and Henry [70] found that PA of PANAS has greater explanatory power for depression than NA in non-clinical samples. Consistent with this evidence, many studies have found that frustration elicited by negative feedback is valid in inducing depression [71-74]. Therefore, in the present study, PA most likely reflects the depression state induced by the frustrating task more sensitively than NA, which may account for the similar NA reports across different groups.

Lastly, one may question that the greater positive affect reduction during the conscious acceptance condition may have an alternative explanation: conscious acceptance group were overtly instructed that "the task is difficult so that they may experience frustration", while this instruction was absent for the other two groups. This might have led to a negative anticipation that enhanced negative feelings. This possibility, however, may not hold true in our study. Firstly, it has been reported that negative anticipation is bound to increased anxiety [75], which is mainly manifested by higher NA scores in PANAS assessment [76]. However, the current study found no significant differences in NA scores between conscious acceptance and the other groups. Secondly, our prior study exploring emotion regulation effect of conscious reappraisal also overtly alerted conscious reappraisal subjects of potential frustration, while this overt instruction was absent for the control subjects. However, it was found that conscious reappraisal did not increase, but significantly reduced the negative emotional experiences in comparison with the control group [44]. These evidences jointly suggest that the largest positive affect reduction during conscious acceptance should be explained by the internal characteristics of conscious acceptance, rather than negative anticipation.

\section{Conclusion}

The present study revealed that both conscious and uncon- scious acceptance strategies significantly decreased emotion-related heart-rate activity. Nevertheless, conscious acceptance had a significant negative subjective emotional consequence, which was absent during unconscious priming of the acceptance strategy. Unconscious acceptance was not linked with a significant reduction in positive affect during the experience of frustration, while this reduction was prominent in the control and conscious acceptance groups. Instructed, conscious acceptance resulted in less positive affect than reported for the control group. This is likely due to the characteristics of conscious acceptance entailing active experience and the full embrace of negative emotional experiences, which may intensify immediate depressive feelings. These effortful processes, however, are unnecessary when priming subjects unconsciously with the acceptance attitude. This suggests that the clinical use of the acceptance strategy may need to consider using unconscious priming of the acceptance strategy, instead of intentionally instructing people to adopt the strategy, for boosting of the efficacy of acceptance in emotion regulation.

This study was supported by the National Natural Science Foundation of China (31170989, 31371042, 31400906), and the Special Grant for Postdoctoral Research in Chongqing (Xm2014059).

1 Davidson K, MacGregor MW, Stuhr J, Dixon K, MacLean D. Constructive anger verbal behavior predicts blood pressure in a population-based sample. Health Psychol, 2000, 19: 55-64

2 Davidson RJ, Putnam KM, Larson CL. Dysfunction in the neural circuitry of emotion regulation - a possible prelude to violence. Science, 2000, 289: 591-594

3 Gross JJ. Emotion regulation in adulthood: timing is everything. Curr Dir Psychol Sci, 2001, 10: 214-219

4 Mayer JD, Salovey P. Emotional intelligence and the construction and regulation of feelings. Appl Prev Psychol, 1995, 4: 197-208

5 Gross JJ. Antecedent-and response-focused emotion regulation: divergent consequences for experience, expression, and physiology. J Pers Soc Psychol, 1998, 74: 224-237

6 John OP, Gross JJ. Individual differences in emotion regulation. In: Gross JJ, eds. Handbook of Emotion Regulation. New York: Guilford Press, 2007. 351-372

7 Moore SA, Zoellner LA, Mollenholt N. Are expressive suppression and cognitive reappraisal associated with stress-related symptoms? Behav Res Ther, 2008, 46: 993-1000

8 Hayes SC, Luoma JB, Bond FW, Masuda A, Lillis J. Acceptance and commitment therapy: model, processes and outcomes. Behav Res Ther, 2006, 44: 1-25

9 Hayes SC, Strosahl KD, Wilson KG. Acceptance and Commitment Therapy: an Experiential Approach to Behavior Change. New York: Guilford Press, 1999

10 Karlin BE, Walser RD, Yesavage J, Zhang A, Trockel M, Taylor CB. Effectiveness of acceptance and commitment therapy for depression: comparison among older and younger veterans. Aging Ment Health, 2013, 17: 555-563

11 McCracken LM, Sato A, Taylor GJ. A trial of a brief group-based form of acceptance and commitment therapy (ACT) for chronic pain in general practice: pilot outcome and process results. J Pain, 2013, 14: 1398-1406

12 Morgan D. Mindfulness-based cognitive therapy for depression: a new approach to preventing relapse. Psychother Res, 2003, 13: $123-125$

13 Day MA, Thorn BE, Ward LC, Rubin N, Hickman SD, Scogin F, 
Kilgo GR. Mindfulness-based cognitive therapy for the treatment of headache pain: a pilot study. Clin J Pain, 2014, 30: 152-161

14 Orsillo SM, Roemer L, Barlow DH. Integrating acceptance and mindfulness into existing cognitive-behavioral treatment for GAD: a case study. Cogn Behav Pract, 2003, 10: 222-230

15 Hayes-Skelton SA, Roemer L, Orsillo SM. A randomized clinical trial comparing an acceptance-based behavior therapy to applied relaxation for generalized anxiety disorder. J Consult Clin Psychol, 2013, 81: 761-773

16 Roemer L, Orsillo SM. An open trial of an acceptance-based behavior therapy for generalized anxiety disorder. Behav Ther, 2007, 38: $72-85$

17 Campbell-Sills L, Barlow DH, Brown TA, Hofmann SG. Acceptability and suppression of negative emotion in anxiety and mood disorders. Emotion, 2006, 6: 587-595

18 Campbell-Sills L, Barlow DH, Brown TA, Hofmann SG. Effects of suppression and acceptance on emotional responses of individuals with anxiety and mood disorders. Behav Res Ther, 2006, 44: 1251-1263

19 Kashdan TB, Barrios V, Forsyth JP, Steger MF. Experiential avoidance as a generalized psychological vulnerability: comparisons with coping and emotion regulation strategies. Behav Res Ther, 2006, 44: $1301-1320$

20 Kashdan TB, Morina N, Priebe S. Post-traumatic stress disorder, social anxiety disorder, and depression in survivors of the Kosovo War: experiential avoidance as a contributor to distress and quality of life. J Anxiety Disord, 2009, 23: 185-196

21 Orcutt HK, Pickett SM, Pope EB. Experiential avoidance and forgiveness as mediators in the relation between traumatic interpersonal events and posttraumatic stress disorder symptoms. J Soc Clin Psychol, 2005, 24: 1003-1029

22 Plumb JC, Orsillo SM, Luterek JA. A preliminary test of the role of experiential avoidance in post-event functioning. J Behav Ther Exp Psychiatry, 2004, 35: 245-257

23 McCracken LM. Learning to live with the pain: acceptance of pain predicts adjustment in persons with chronic pain. Pain, 1998, 74: $21-27$

24 Shallcross AJ, Troy AS, Boland M, Mauss IB. Let it be: accepting negative emotional experiences predicts decreased negative affect and depressive symptoms. Behav Res Ther, 2010, 48: 921-929

25 Lang PJ, Bradley MM, Cuthbert BN. Motivated attention: affect, activation, and action. In: Lang PJ, Simons RF, Balaban M, eds. Attention and Orienting: Sensory and Motivational Processes. Mahwah: Lawrence Erlbaum Associates, 1997. 97-135

26 Yuan J, Long Q, Ding N, Lou Y, Liu Y, Yang J. Suppression dampens unpleasant emotion faster than reappraisal: neural dynamics in a Chinese sample. Sci China Life Sci, 2015, 58: 480-491

27 Dunn BD, Billotti D, Murphy V, Dalgleish T. The consequences of effortful emotion regulation when processing distressing material: a comparison of suppression and acceptance. Behav Res Ther, 2009, 47: $761-773$

28 Hofmann SG, Heering S, Sawyer AT, Asnaani A. How to handle anxiety: the effects of reappraisal, acceptance, and suppression strategies on anxious arousal. Behav Res Ther, 2009, 47: 389-394

29 Rood L, Roelofs J, Bögels SM, Arntz A. The effects of experimentally induced rumination, positive reappraisal, acceptance, and distancing when thinking about a stressful event on affect states in adolescents. J Abnorm Child Psychol, 2012, 40: 73-84

30 Mauss IB, Bunge SA, Gross JJ. Automatic emotion regulation. Soc Personal Psychol Compass, 2007, 1: 146-167

31 Bonanno GA, Papa A, Lalande K, Westphal M, Coifman K. The importance of being flexible the ability to both enhance and suppress emotional expression predicts long-term adjustment. Psychol Sci, 2004, 15: 482-487

32 Gross JJ, John OP. Individual differences in two emotion regulation processes: implications for affect, relationships, and well-being. J Pers Soc Psychol, 2003, 85: 348-362

33 Polivy J. The effects of behavioral inhibition: integrating internal cues, cognition, behavior, and affect. Psychol Inq, 1998, 9: 181-204
34 Butler EA, Lee TL, Gross JJ. Emotion regulation and culture: are the social consequences of emotion suppression culture-specific? Emotion, 2007, 7: 30-48

35 Mauss IB, Cook CL, Gross JJ. Automatic emotion regulation during anger provocation. J Exp Soc Psychol, 2007, 43: 698-711

36 Carstensen LL, Mikels JA. At the intersection of emotion and cognition aging and the positivity effect. Curr Dir Psychol Sci, 2005, 14: $117-121$

37 Isaacowitz DM, Wadlinger HA, Goren D, Wilson HR. Is there an age-related positivity effect in visual attention? A comparison of two methodologies. Emotion, 2006, 6: 511-516

38 Bargh JA, Gollwitzer PM, Lee-Chai A, Barndollar K, Trötschel R. The automated will: nonconscious activation and pursuit of behavioral goals. J Pers Soc Psychol, 2001, 81: 1014-1027

39 Chartrand TL, Jefferis VE. Consequences of automatic goal pursuit and the case of nonconscious mimicry. In: Forgas JP, Williams KD, von Hippel W, eds. Social Judgments: Implicit and Explicit Processes. New York: Cambridge University Press, 2003. 290-305

40 Fitzsimons GM, Bargh JA. Automatic self-regulation. In: Baumeister R F, Vohs K D, eds. Handbook of Self-regulation: Research, Theory, and Applications. New York: Guilford Publications, 2004. 151-170

41 Koole SL, Jostmann NB. Getting a grip on your feelings: effects of action orientation and external demands on intuitive affect regulation. J Pers Soc Psychol, 2004, 87: 974-990

42 Koole SL, Rothermund K. "I feel better but I don't know why": the psychology of implicit emotion regulation. Cogn Emot, 2011, 25: 389-399

43 Gallo IS, Keil A, McCulloch KC, Rockstroh B, Gollwitzer PM. Strategic automation of emotion regulation. J Pers Soc Psychol, 2009, 96: $11-31$

44 Yuan J, Ding N, Liu Y, Yang J. Unconscious emotion regulation: nonconscious reappraisal decreases emotion-related physiological reactivity during frustration. Cogn Emot, 2014, doi: 10.1080/ 02699931.2014 .965663

45 Eder $\mathrm{AB}$. Control of impulsive emotional behaviour through implementation intentions. Cogn Emot, 2011, 25: 478-489

46 Washington G, Adviser-Jones RP. Understanding the impact of user frustration intensities on task performance using a novel adaptation of the occ theory of emotions. Doctor Dissertation. Washington: The George Washington University, 2011

47 Williams LE, Bargh JA, Nocera CC, Gray JR. The unconscious regulation of emotion: nonconscious reappraisal goals modulate emotional reactivity. Emotion, 2009, 9: 847-854

48 Gross JJ, Levenson RW. Hiding feelings: the acute effects of inhibiting negative and positive emotion. J Abnorm Psychol, 1997, 106: 95-103

49 Jost H. Some physiological changes during frustration. Child Dev, 1941, 12: 9-15

50 Spielberger CD. STAI manual for the state-trait anxiety inventory. Self-Evaluation Questionnaire. Palo Alto: Consulting Psychologists, 1970. $1-24$

51 Beck A, Steer R, Brown G. BDI-II, Beck depression inventory. Manual: Psychological Corp. San Antonio, 1996

52 Rosenberg M. Rosenberg Self-Esteem Scale (RSE). Acceptance and Commitment Therapy. Measures Package. New York: Basic Books, 1979. 61

53 Hayes SC, Strosahl KD, Wilson KG, Bissett RT, Pistorello J, Toarmino D, Polusny MA, Dykstra TA, Batten SV, Bergan J, Stewart SH, Zvolensky MJ, Eifert GH, Bond FW, Forsyth J P, Karekla M, McCurry SM. Measuring experiential avoidance: a preliminary test of a working model. Psychol Rec, 2004, 54: 553-578

54 Srull TK, Wyer RS. The role of category accessibility in the interpretation of information about persons: some determinants and implications. J Pers Soc Psychol, 1979, 37: 1660-1672

55 Bargh JA, Chartrand TL. The mind in the middle: a practical guide to priming and automaticity research. In: Reis HT, Judd CM, eds. Handbook of Research Methods in Social and Personality Psychology. New York: Cambridge University Press, 2000. 253-285

56 Watson D, Clark LA, Tellegen A. Development and validation of 
brief measures of positive and negative affect: the PANAS scales. J Pers Soc Psychol, 1988, 54: 1063-1070

57 Grèzes J, Philip L, Chadwick M, Dezecache G, Soussignan R, Conty L. Self-relevance appraisal influences facial reactions to emotional body expressions. PLoS One, 2013, 8: e55885

58 Phan KL, Taylor SF, Welsh RC, Ho SH, Britton JC, Liberzon I. Neural correlates of individual ratings of emotional salience: a trial-related fMRI study. Neuroimage, 2004, 21: 768-780

59 Yang J, Yuan J, Li H. Expectation decreases brain susceptibility to fearful stimuli: ERP evidence from a modified emotion evaluation task. Neurosci Lett, 2012, 514: 198-203

60 Liverant GI, Brown TA, Barlow DH, Roemer L. Emotion regulation in unipolar depression: the effects of acceptance and suppression of subjective emotional experience on the intensity and duration of sadness and negative affect. Behav Res Ther, 2008, 46: 1201-1209

61 Eifert GH, Heffner M. The effects of acceptance versus control contexts on avoidance of panic-related symptoms. J Behav Ther Exp Psychiatry, 2003, 34: 293-312

62 Levitt JT, Brown TA, Orsillo SM, Barlow DH. The effects of acceptance versus suppression of emotion on subjective and psychophysiological response to carbon dioxide challenge in patients with panic disorder. Behav Ther, 2004, 35: 747-766

63 Kuehner C, Huffziger S, Liebsch K. Rumination, distraction and mindful self-focus: effects on mood, dysfunctional attitudes and cortisol stress response. Psychol Med, 2009, 39: 219-228

64 Kabat-Zinn J. Mindfulness-based interventions in context: past, present, and future. Clin Psychol: Sci Pract, 2003, 10: 144-156

65 Segal ZV, Williams JMG, Teasdale JD. Mindfulness-based Cognitive Therapy for Depression. New York: Guilford Press, 2012

66 Larsen RJ, Ketelaar T. Personality and susceptibility to positive and negative emotional states. J Pers Soc Psychol, 1991, 61: 132-140
67 Kahneman D, Diener E, Schwarz N. Well-being: Foundations of Hedonic Psychology. New York: Russell Sage Foundation, 2003

68 Dyck MJ, Jolly JB, Kramer T. An evaluation of positive affectivity, negative affectivity, and hyperarousal as markers for assessing between syndrome relationships. Pers Individ Dif, 1994, 17: 637-646

69 Jolly JB, Dyck MJ, Kramer TA, Wherry JN. Integration of positive and negative affectivity and cognitive content-specificity: improved discrimination of anxious and depressive symptoms. J Abnorm Psychol, 1994, 103: 544-552

70 Crawford JR, Henry JD. The positive and negative affect schedule (PANAS): construct validity, measurement properties and normative data in a large non-clinical sample. Brit J Clin Psychol, 2004, 43: 245-265

71 Nummenmaa L, Niemi P. Inducing affective states with successfailure manipulations: a meta-analysis. Emotion, 2004, 4: 207-214

72 Baars M, Müller MJ, Gallhofer B, Netter P. Depressive and aggressive responses to frustration: development of a questionnaire and its validation in a sample of male alcoholics. Depress Res Treat, 2011, 2011: 352048

73 Westermann R, Spies K, Stahl G, Hesse FW. Relative effectiveness and validity of mood induction procedures: a meta-analysis. Eur J Soc Psychol, 1996, 26: 557-580

74 Goodwin AM, Williams JMG. Mood-induction research-its implications for clinical depression. Behav Res Ther, 1982, 20: 373-382

75 MacLeod AK, Byrne A. Anxiety, depression, and the anticipation of future positive and negative experiences. J Abnorm Psychol, 1996, 105: 286-289

76 Ebesutani C, Okamura K, Higa-McMillan C, Chorpita BF. A psychometric analysis of the Positive and Negative Affect Schedule for Children-Parent Version in a school sample. Psychol Assess, 2011, 23: 406-416

Open Access This article is distributed under the terms of the Creative Commons Attribution License which permits any use, distribution, and reproduction in any medium, provided the original author(s) and source are credited. 